\title{
Code pairs with specified parity of the Hamming distances
}

\author{
R. Ahlswede*, Z. Zhang \\ Universitaet Bielefeld, Facultaet fur Mathematik., Postfach 100131. D-33501 Bielefeld, Germany:
}

Received 13 August 1996; revised 28 October 1997; accepted 17 October 1997

\begin{abstract}
For code pairs $(A, B) ; A, B \subset\{0,1, \ldots, \alpha-1\}^{n}$; with mutually constant parity of the Hamming distances a conjecture of the first author concerning the maximal value of $|A||B|$ is established. (c) 1998 Elsevier Science B.V. All rights reserved

Keywords: Constant distance pair of codes; Parity of the Hamming distances; Monochromatic rectangles; Extremal problems
\end{abstract}

\section{Introduction and results}

Constant distance codes have been investigated in [7,9]. The study of pairs of codes with mutually constant distances was initiated in [3] and is continued in Refs. $[4,5]$. Weakening of the constant distance property led via $[4,5]$ to the quite general 4-words inequality of [2]. In another direction, in [1] constant distance code pairs where analysed for specified distances and also for non-binary alphabets. There, also extremal problems with constant parity of the Hamming distance were considered. We quickly report the results and conjectures.

$\mathscr{X}_{\alpha}=\{0,1, \ldots, \alpha-1\}$ is a finite set or alphabet. The pair $(A, B)$ with $A, B \subset \mathscr{X}_{\alpha}^{n}=$ $\prod_{1}^{n} \mathscr{X}_{x}$ is called an $(n, \delta)$-system (or constant distance code pair with parameters $n, \delta$ ), if for the Hamming distance function $d$

$$
d(a, b)=\delta \text { for all } a \in A, b \in B .
$$

Let $\mathscr{S}_{\alpha}(n, \delta)$ denote the set of those systems and set

$$
\begin{aligned}
& M_{\alpha}(n, \delta)=\max \left\{|A||B|:(A, B) \in \mathscr{P}_{\alpha}(n, \delta)\right\}, \\
& M_{\alpha}(n)=\max _{0 \leqslant \delta \leqslant n} M_{\alpha}(n, \delta) .
\end{aligned}
$$

The discovery of [3] was

* Corresponding author. E-mail: hollmann@mathematik.uni-bielefeld.de. 


\section{Theorem AGP.}

$$
M_{2}(n)= \begin{cases}2^{n}, & \text { if } n \text { is even } \\ 2^{n-1}, & \text { if } n \text { is odd }\end{cases}
$$

Next, in [9] $M_{\chi}(n, \delta)$ has been related to the functions $F_{\chi}(n, \delta)$, where

$$
\begin{aligned}
& F_{2}(n, \delta)=\max _{\delta_{1}+\delta_{2}=\delta}(4)^{\delta_{1}}\left(\begin{array}{c}
n-2 \delta_{1} \\
\delta_{2}
\end{array}\right), \quad 4=2 \cdot 2 ! \\
& F_{3}(n, \delta)=\max _{2 \delta_{1}+\delta_{2}=\delta}(18)^{\delta_{1}}\left(\begin{array}{c}
n-3 \delta_{1} \\
\delta_{2}
\end{array}\right) 2^{\delta_{2}}, \quad 18=3 \cdot 3 ! \\
& F_{\alpha}(n, \delta)=\max _{\delta_{1}+\delta_{2}=\delta}\left(\left\lfloor\frac{\alpha}{2}\right\rfloor\left[\frac{\alpha}{2}\right\rceil\right)^{\delta_{1}}\left(\begin{array}{c}
n-\delta_{1} \\
\delta_{2}
\end{array}\right)(\alpha-1)^{\delta_{2}} \text { for } \alpha \geqslant 4 .
\end{aligned}
$$

Theorem $\mathbf{A}_{1}$. For $n \in \mathbb{N}, 0 \leqslant \delta \leqslant n$

(i) $M_{2}(n, \delta)=F_{2}(n, \delta)$.

(ii) $M_{x}(n, \delta)=F_{x}(n, \delta)$ for $\alpha=4,5$.

\section{Conjecture $\mathbf{A}_{l}$.}

(iii) $M_{3}(n, \delta)=F_{3}(n, \delta)$.

(iv) $M_{\alpha}(n, \delta)=F_{\alpha}(n, \delta)$ for $\alpha \geqslant 6$.

Finally we come to the subject of this paper, namely, code pairs with a parity constraint. It is convenient to introduce the function $\Psi: \mathbb{N} \cup\{0\} \rightarrow\{0,1\}$, where

$$
\Psi(n)= \begin{cases}0, & \text { if } n \text { is even }, \\ 1, & \text { if } n \text { is odd }\end{cases}
$$

We consider the parity function $\Pi: \bigcup_{n=1}^{\infty}\left(\mathscr{X}_{\alpha}^{n} \times \mathscr{X}_{\alpha}^{n}\right) \rightarrow\{0,1\}$ defined by

$$
\Pi\left(x^{n}, y^{n}\right)=\Psi\left(d\left(x^{n}, y^{n}\right)\right) .
$$

The pair $(A, B)$ with $A, B \in \mathscr{X}_{\alpha}^{n}$ is said to have $p$-parity, if

$$
\Pi(a, b)=p \text { for all } a \in A, b \in B .
$$

For $p=0,1$ let $\mathscr{P}_{\alpha}^{\prime \prime}(x)$ denote the set of those $p$-parity pairs and define

$$
\begin{aligned}
& Q_{\alpha}^{p}(n)=\max \left\{|A||B|:(A, B) \in \mathscr{P}_{\alpha}^{\prime \prime}(n)\right\}, \\
& Q_{\alpha}(n)=\max _{p=0,1} Q_{\alpha}^{p}(n) .
\end{aligned}
$$

This last quantity is known for all $n$ and $\alpha \neq 3$, and $Q_{x}^{p}(n)$ is almost known.

Theorem $\mathbf{A}_{2}$ (Ahlswede [1]). For $n \in \mathbb{N}$ and $\bar{\alpha}=\left\lfloor\frac{\alpha}{2}\right\rfloor \cdot\left\lceil\frac{\alpha}{2}\right\rceil$

(a) $Q_{x}^{p}(n)=\bar{\alpha}^{n}$, if $\Psi(n)=p(\alpha \geqslant 4 ; p=0,1)$,

$\left(\mathrm{a}^{\prime}\right) \bar{\alpha}^{n-1} \leqslant Q_{x}^{p}(n)<\bar{\alpha}^{n}$, if $\Psi(n) \neq p(\alpha \geqslant 4 ; p=0,1)$, 
$\left(\mathrm{a}^{\prime \prime}\right) Q_{\alpha}(n)=\bar{\alpha}^{n}(\alpha \geq 4)$,

(b) $Q_{2}(n)=Q_{2}^{0}(n)=Q_{2}^{1}(n)=4^{n-1}$.

For $\alpha=3$ we have

\section{Conjecture $\mathbf{A}_{2}$.}

(c) $Q_{3}^{p}(n)=\left(2^{n-1}+1\right)\left(2^{n-1}+1\right)$, if $\Psi(n)=p=0$.

(c $\left.\mathrm{c}^{\prime}\right) Q_{3}^{p}(n)=\left(2^{n-1}+1\right) 2^{n-1}$, if $\Psi(n)=p=1$.

(c $\left.{ }^{\prime \prime}\right) Q_{3}^{p}(n)=2^{n-1} \cdot 2^{n-1}$, if $\Psi(n) \neq p$ and $n \neq 3$.

In the exceptional case $n=3, p=0$ not covered, one readily verifies that

$$
(A, B)=(\{111,222,333\}, \quad \text { all permutations of } 123\})
$$

is optimal and that therefore $Q_{3}^{0}(3)=18$. A first insight can be gained from the following key tool of [1]. For $B \subset \mathscr{X}_{2}^{n}$ and $T \subset\{1,2, \ldots, n\}$ we say that $B$ has parity on $T$, if the projection $\operatorname{Proj}_{T} B$ on $\prod_{t \in T} \mathscr{X}_{2}$ contains only sequences with an odd or only sequences with an even number of ones.

Lemma (Blockwise parity property)

$$
\sum_{\substack{T \subset\{1,2, \ldots, n\} \\ B \text { has parity on } T}} 2^{|T|}|B| \leqslant\left(2^{n}+1\right) 2^{n-1} \text { for every } B \subset X_{2}^{n} .
$$

The right-hand bound is assumed, for instance, if $B$ equals the set of all sequences with an even number of ones. The result of this paper is

Theorem. Conjecture $A_{2}$ is true.

Finally, we draw attention to an open problem. For single sets, $A$ has $p$-parity, if

$$
\Pi\left(a, a^{\prime}\right)=p \quad \text { for } a, a^{\prime} \in A \text { with } a \neq a^{\prime} .
$$

The quantity $q_{x}^{p}(n)=\max \left\{|A|: A \subset \mathscr{X}_{x}^{n}\right.$ has $p$-parity $\}$ has been determined in [1] for $p=0$ (and all values for $\alpha$ and $n$ ). There are only bounds for $q_{x}^{1}(n)$. Determine $q_{x}^{\mathrm{l}}(n)$ !

\section{Proof of Theorem: the direct part}

Our alphabet is $\mathscr{X}_{3}=\{0,1,2\}$. Let us define

$$
\langle a \mid x\rangle=\text { number of occurrences of letter } x \text { in word } a .
$$

We need the sets

$$
\mathscr{E}_{2}(n)=\left\{a \in \mathscr{X}_{2}^{n}:\langle a \mid 1\rangle \text { is even }\right\}, \quad \mathscr{O}_{2}(n)=\left\{a \in \mathscr{X}_{2}^{n}:\langle a \mid 1\rangle \text { is odd }\right\},
$$


and the word

$$
\underline{2}=(2,2, \ldots, 2) \in X_{3}^{n} \text {. }
$$

We show first how the values for $Q_{3}^{p}(n)$ specified in Conjecture $A_{2}$ can be achieved. For this choose

(c) $(A, B)=\left(\mathscr{E}_{2}(n) \cup\{\underline{2}\}, \mathscr{E}_{2}(n) \cup\{\underline{2}\}\right)$

(c') $(A, B)=\left(\mathscr{E}_{2}(n) \cup\{\underline{2}\}, \mathscr{O}_{2}(n)\right)$ or $(A, B)=\left(\mathscr{E}_{2}(n), \mathcal{O}_{2}(n) \cup\{\underline{2}\}\right)$

$\left(\mathrm{c}^{\prime \prime}\right)(A, B)=\left(\mathscr{E}_{2}(n), \mathscr{E}_{2}(n)\right)$ or $\left(\mathscr{O}_{2}(n), \mathcal{O}_{2}(n)\right)$, if $p=0$, and $(A, B)=\left(\mathcal{O}_{2}(n), \mathscr{E}_{2}(n)\right.$, if $p=1$.

\section{Proof of Theorem: the converse part}

\subsection{Basic concepts and their properties}

For $A, B \subset \mathscr{X}_{3}^{n}$ define

$$
A_{s t}^{i j}=\left\{x^{n-2} \in \prod_{\substack{l \neq i, j \\ 1 \leqslant l \leqslant n}} \mathscr{X}_{3}:\left(x_{1}, \ldots, x_{i-1}, s, x_{i+1}, \ldots, x_{j-1}, t, x_{j+1}, \ldots, x_{n}\right) \in A\right\}
$$

and similarly define $B_{s t}^{i j}$. For simplicity, we consider $A_{s t}^{12}, B_{s t}^{12}$ and denote them by $A_{s t}$ and $B_{s t}$, respectively. Define

$$
I=\left\{\left\{\left(\begin{array}{l}
s_{1} t_{1} \\
s_{2} t_{2}
\end{array}\right)\right\}: A_{s_{1}, t_{1}} \cap A_{s_{2}, t_{2}} \neq \emptyset\right\} .
$$

For

$$
a=\left(\begin{array}{l}
s_{1} t_{1} \\
s_{2} t_{2}
\end{array}\right)
$$

define

$$
\begin{aligned}
& \mathscr{P}(a)=\left\{(s, t):(s, t) \text { has the same parity with both }\left(s_{1}, t_{1}\right) \text { and }\left(s_{2}, t_{2}\right)\right\}, \\
& \mathscr{S}(I)=\bigcap_{a \in I} \mathscr{P}(a) .
\end{aligned}
$$

Similarly define $J, \mathscr{S}(b)$, and $\mathscr{S}(J)$. Now, if $(A, B)$ has $p$-parity these sets must have the following properties

(1) $\left\{\left(s_{1}, t_{1}\right),\left(s_{2}, t_{2}\right)\right\} \in I \Rightarrow\left(s_{1}, t_{1}\right),\left(s_{2}, t_{2}\right) \in \mathscr{T}(J)$,

$\left\{\left(s_{1}, t_{1}\right),\left(s_{2}, t_{2}\right)\right\} \in J \Rightarrow\left(s_{1}, t_{1}\right),\left(s_{2}, t_{2}\right) \in \mathscr{S}(I)$,

(2) $(s, t) \notin \mathscr{S}(I) \Rightarrow B_{s t}=\emptyset$,

$(s, t) \notin \mathscr{P}(j) \Rightarrow A_{s t}=\emptyset$. 
A pair $(I, J)$, which satisfies these properties, is called matching, and $(\mathscr{S}(I), \mathscr{S}(J))$ is called proper. If $\left(I_{1}, J_{1}\right),\left(I_{2}, J_{2}\right)$ are two matching pairs, and $\mathscr{S}\left(I_{1}\right)=\mathscr{S}\left(I_{2}\right), \mathscr{S}\left(J_{1}\right)=$ $\mathscr{S}\left(J_{2}\right)$, then $\left(I_{1} \cup I_{2}, J_{1} \cup J_{2}\right)$ is also a matching pair, and

$$
\mathscr{P}\left(I_{1} \cup I_{2}\right)=\mathscr{S}\left(I_{1}\right)=\mathscr{S}\left(I_{2}\right), \quad \mathscr{S}\left(J_{1} \cup J_{2}\right)=\mathscr{S}\left(J_{1}\right)=\mathscr{S}\left(J_{2}\right) .
$$

This means that given a proper pair $(\mathscr{S}(I), \mathscr{S}(J))$, there exists a maximal matching pair $(I, J)$ reaching this proper pair. Any other matching pair $\left(I^{\prime}, J^{\prime}\right)$ such that $\mathscr{S}\left(I^{\prime}\right)=$ $\mathscr{S}(I), \mathscr{S}\left(J^{\prime}\right)=\mathscr{S}(J)$ satisfies

$$
I^{\prime} \subset I, \quad J^{\prime} \subset J .
$$

Now we explain why we define these concepts. We are going to use induction to prove the conjecture. We need to divide $\mathscr{S}(I) \times \mathscr{P}(J)$ into smaller rectangles such that for each rectangle

$$
\left\{\left(s_{11}, t_{11}\right), \ldots,\left(s_{1 m}, t_{1 m}\right)\right\} \times\left\{\left(s_{21}, t_{21}\right), \ldots,\left(s_{2 k}, t_{2 k}\right)\right\}
$$

$\left\{\left(s_{1 i}, t_{1 i}\right),\left(s_{2 j}, t_{2 j}\right)\right\}$ should have the same parity. Denote the number of such rectangles, which have parity 0 , by $\alpha$, and the number of such rectangles, which have parity 1 , by $\beta$. These rectangles cover the whole $\mathscr{S}(I) \times \mathscr{S}(J)$. Therefore we obtain

$$
Q_{3}^{p}(n) \leqslant \alpha Q_{3}^{p}(n-2)+\beta Q_{3}^{\bar{p}}(n-2)
$$

where $\bar{p}=1+p \bmod 2$. But these rectangles must have the property that

$$
\left\{\left(s_{1 i}, t_{1 i}\right),\left(s_{1 j}, t_{1 j}\right)\right\} \notin I \quad \text { and } \quad\left\{\left(s_{2 i}, t_{2 i}\right),\left(s_{2 j}, t_{2 j}\right)\right\} \notin J,
$$

because for the pairs $\left(s_{1}, t_{1}\right),\left(s_{2}, t_{2}\right)$ in $I$

$$
A_{s_{1}, t_{1}} \cap A_{s_{2}, t_{2}} \neq \emptyset \text {. }
$$

For the maximal matching pair we denote the corresponding $\alpha$ and $\beta$ by $\alpha(\mathscr{S}(I), \mathscr{S}(J))$ and $\beta(\mathscr{S}(I), \mathscr{S}(J))$. Then any other matching pair $\left(I^{\prime}, J^{\prime}\right)$, which has the same proper pair $(\mathscr{S}(I), \mathscr{S}(J))$, must satisfy

$$
\alpha \leqslant \alpha(\mathscr{P}(I), \mathscr{S}(J)), \quad \beta \leqslant \beta(\mathscr{S}(I), \mathscr{S}(J)) .
$$

This means that for the induction we need to consider only the maximal matching pairs.

\subsection{Determination of all the proper pairs and their corresponding maximal matching pairs}

Lemma 1. We have the following maximal matching pairs:

(1) $I=\emptyset, J=\emptyset, \mathscr{S}(I)=\mathscr{S}(J)=\Omega \triangleq \mathscr{X}_{3}^{2}$.

(2) $I=\{(00,11),(01,10),(02,20),(00,22),(00,12),(00,21),(11,21),(11,12),(12,21)$, $(22,12),(22,21),(11,22),(01,02),(01,20),(02,10),(10,20)\}$, $J=\emptyset, S(I)=\{(00)\}, S(J)=\Omega$. 
(3) $I=\{(22,12),(00,11),(00,21),(11,21),(01,10),(01,20),(10,20)\}$, $J=\emptyset, \mathscr{S}(I)=\{(00),(01)\}, \mathscr{S}(J)=\Omega$.

(4) $J=\emptyset, \mathscr{S}(J)=\Omega, I=\{(00,11),(00,22),(11,22),(01,10),(02,20),(12,21)\}$, $\mathscr{S}(I)=\{(00),(11),(22)\}$.

(5) $I=J=\{(00,11),(00,22),(11,22)\}, \mathscr{S}(I)=\mathscr{S}(J)=\{(00),(11),(22)\}$.

(6) $J=\{(00,11)\}, I=\{(00,11),(00,22),(11,22),(01,10)\}$, $\mathscr{S}(I)=\{(00),(11),(22),(01),(10)\}, \mathscr{S}(J)=\{(00),(11),(22)\}$.

(7) $I=\{(00,01),(10,11),(20,21)\}, J=\emptyset$, $\mathscr{S}(I)=\{(02),(12),(22)\}, \mathscr{S}(J)=\Omega$.

(8) $I=\{(20,21)\}, J=\{(02),(12)\}$, $\mathscr{S}(I)=\{(02),(12),(22)\}, \mathscr{S}(J)=\{(20),(21),(22)\}$.

(9) $J=\emptyset, I=\{(00,11),(01,10)\}, \mathscr{P}(J)=\Omega, \mathscr{S}(I)=\{(00),(11),(22),(01),(10)\}$.

(10) $I=J=\{(00,11),(01,10)\},, \mathscr{S}(I)=\mathscr{S}(J)=\{(00),(11),(22),(01),(10)\}$.

Proof. We search for the maximal matching pairs in the following procedure. First, obviously $(\emptyset, \emptyset)$ should be such a pair and it corresponds to the proper pair $(\Omega, \Omega)$, where

$$
\Omega=\{(00),(01),(10),(11),(02),(20),(12),(21),(22)\} .
$$

For other cases we assume $I \neq \emptyset$. Without loss of generality we can assume that either $\{(00),(11)\} \in I$ or $\{(00),(01)\} \in I$, because other cases are equivalent to one of these two cases.

Now if $\{(00),(11)\} \in I$, then $\mathscr{P}(I) \subset\{(00),(11),(22),(01),(10)\}$, and if $\{(00),(01)\} \in I, \quad$ then $\mathscr{S}(I) \subset\{(02),(12),(22)\}$.

Subcase $\min \{|\mathscr{S}(I)|,|\mathscr{S}(J)|\}=1$. There is only one class, that is $\mathscr{P}(I)=\{(00)\}$, $J=\emptyset$, and

$$
\begin{aligned}
I=\{ & (00,11),(01,10),(02,20),(00,22),(00,12),(00,21),(11,21),(11,12), \\
& (12,21),(22,12),(22,21),(11,22),(01,02),(01,20),(02,10),(10,20)\} .
\end{aligned}
$$

Subcase $\min \{|\mathscr{S}(I)|,|\mathscr{S}(J)|\}=2$. There are two possibilities: (a) $\mathscr{S}(I)=\{(00),(11)\}$ and (b) $\mathscr{S}(I)=\{(00),(01)\}$.

If $J=\emptyset$, then in case (a) $I=\{(00,11),(00,22),(11,22),(01,10),(02,20),(12,21)\}$. These are all the pairs $a$ with

$$
\mathscr{S}(a) \supset\{(00),(11)\} \quad \text { but } \mathscr{S}(I)=\{(00),(11),(22)\} .
$$

Therefore $(\Omega,\{(00),(11)\})$ is not a proper pair. There is no maximal matching pair in case (a) even if $J \neq \emptyset$. In case (b) $I=\{(22,12),(00,11),(00,21),(11,21),(01,12)$, $(01,20),(10,20)\}$. This is a maximal matching pair. If $|J|=1$, then $J=\{(00,01)\}$, $\mathscr{S}(J)=\{02,12,22\}, I=\{(22,12)\}$, but $\mathscr{S}(I)=\{00,01,02\} \neq\{00,01\}$. This means that there is no maximal matching pair in this case. 
Subcase $\min \{|\mathscr{S}(I)|,|\mathscr{S}(J)|\}=3$ There are the following possibilities:
(a) $\mathscr{S}(J)=\{00,11,22\}$,
(b) $\mathscr{S}(I)=\{00,01,11\}$,
(c) $\mathscr{S}(J)=\{01,11,22\}$,
(d) $\mathscr{S}(J)=\{02,12,22\}$.

It is easy to check that in cases (b) and (c) there is no maximal matching pair, so we consider only (a) and (d).

In case (a) there are only 3 maximal matching pairs, namely

$$
\begin{aligned}
& I=\{(00,11),(00,22),(11,22),(01,10),(02,20),(12,21)\}, \quad J=\emptyset, \\
& I=J=\{(00,11),(00,22),(11,22)\}, \quad I=\{(00,11),(00,22),(11,22),(01,10)\} \\
& J=\{(00,11)\} .
\end{aligned}
$$

In case $(\mathrm{d}), J=\emptyset$ and then $I=\{(00,01),(10,11),(20,21)\}$. This is a maximal matching pair. If $|J|=1$, let $J=\{(02,12)\}$. Then, $\mathscr{S}(J)=\{20,21,22\}$ and $I=$ $\{(20,21)\}$. This is a maximal matching pair. In case $\min \{|I|,|J|\}>1$, there is no maximal matching pair.

Subcase $\min \{|S(I)|,|\mathscr{S}(J)|\}=4$. There is no maximal matching pair, because for any two pairs $a$ and $b,|\mathscr{S}(a) \cap \mathscr{S}(b)|=5,3$, or 2 .

Subcase $\min \{|\mathscr{S}(I)|,|\mathscr{S}(J)|\}=5 . \mathscr{S}(I)=\{(00),(11),(22),(01),(10)\}, J=\emptyset$, $I=\{(00,11),(01,10)\}$ or $J \neq \emptyset$ and the maximal matching is $I=J=\{(00,11)$, $(01,10)\}$. These are all of the maximal matching pairs.

\subsection{The coefficients $\alpha, \beta$ for each of the ten maximal matching pairs}

1. We use the parity table, Table 1. From the parity table we take the following 6 squares

$$
\left\{\left(0 t_{0}\right),\left(1 t_{1}\right),\left(2 t_{2}\right)\right\} \times\left\{\left(0 t_{0}\right),\left(1 t_{1}\right),\left(2 t_{2}\right)\right\},
$$

Table 1

\begin{tabular}{llllllllll}
\hline & 00 & 01 & 10 & 11 & 02 & 20 & 12 & 21 & 22 \\
\hline 00 & 0 & 1 & 1 & 0 & 1 & 1 & 0 & 0 & 0 \\
01 & 1 & 0 & 0 & 1 & 1 & 0 & 0 & 1 & 0 \\
10 & 1 & 0 & 0 & 1 & 0 & 1 & 1 & 0 & 0 \\
11 & 0 & 1 & 1 & 0 & 0 & 0 & 1 & 1 & 0 \\
02 & 1 & 1 & 0 & 0 & 0 & 0 & 1 & 0 & 1 \\
20 & 1 & 0 & 1 & 0 & 0 & 0 & 0 & 1 & 1 \\
12 & 0 & 0 & 1 & 1 & 1 & 0 & 0 & 0 & 1 \\
21 & 0 & 1 & 0 & 1 & 0 & 1 & 0 & 0 & 1 \\
22 & 0 & 0 & 0 & 0 & 1 & 1 & 1 & 1 & 0 \\
\hline
\end{tabular}


Table 2

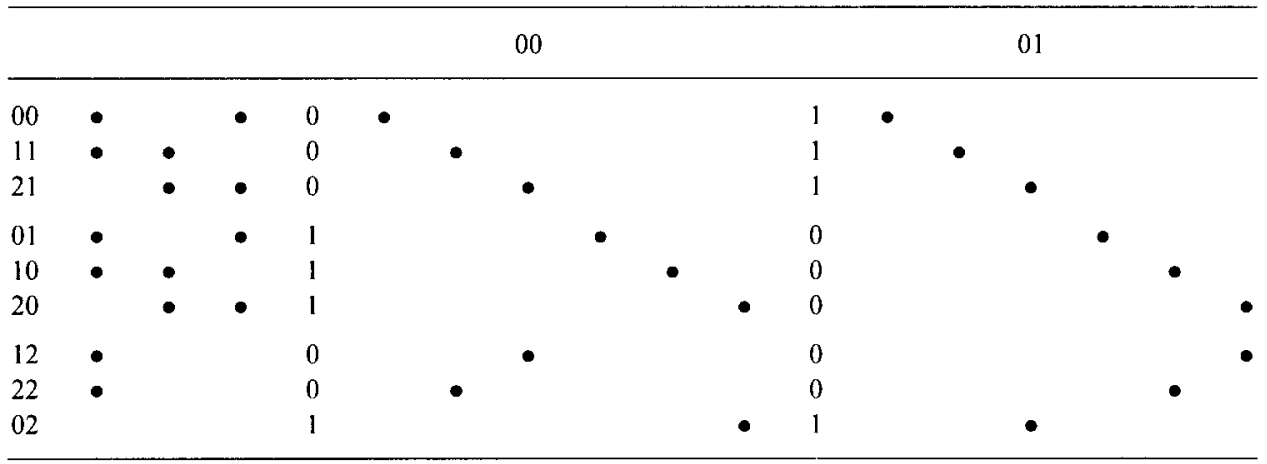

Table 3

00

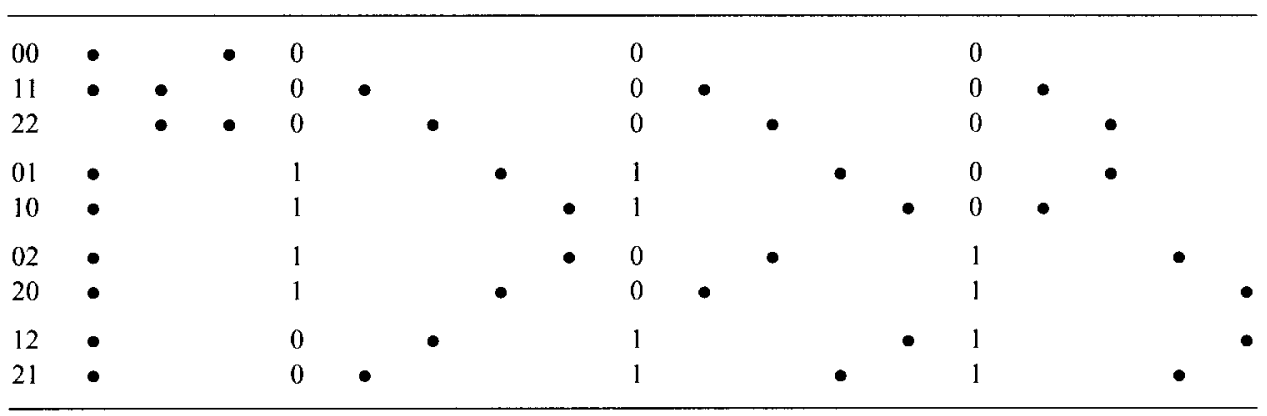

and additionally the hyperedge $\{(00,00),(00,11),(00,22)\}$

Table 4
11

22

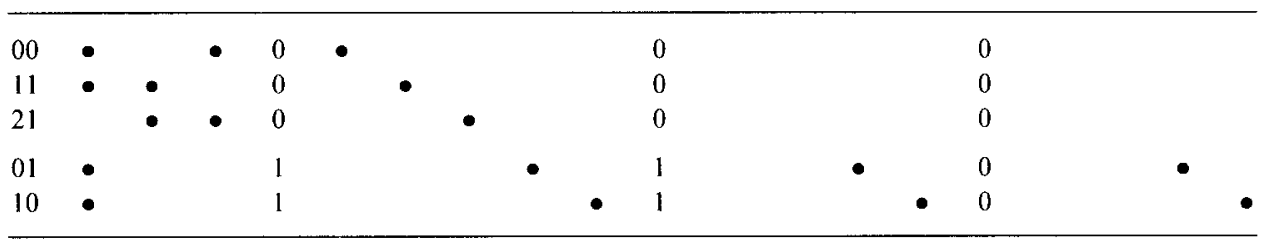

and additionally the hyperedges

$\{(00,11),(00,22)\},\{(11,11),(11,22)\},\{(22,11),(22,22)\}$ and $(00,11)$ is a pair in $J$.

where $\left(t_{0}, t_{1}, t_{2}\right)$ is a permutation of $(0,1,2)$. All of them have parity 0 and they cover all 0 's in the table. Further, the 9 rectangles $\{(t, s)\} \times\{(i, j):(i, j)$ has parity 1 with $(t, s)\}$ cover all 1 's. Thus $\alpha \leqslant 6, \beta \leqslant 9$.

2. $|\mathscr{P}(I)|=1$ and thus $\alpha+\beta \leqslant 9$. 
Table 5

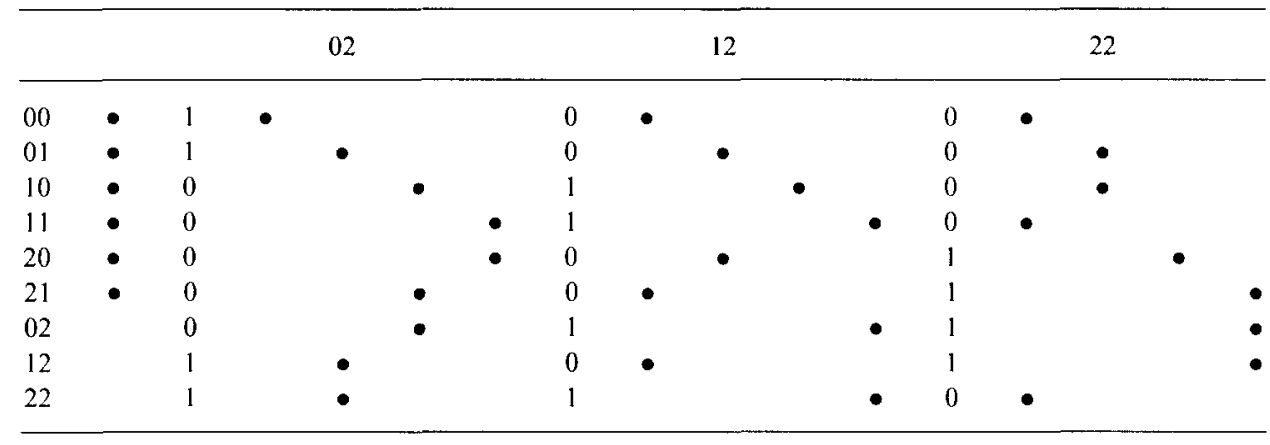

Table 6

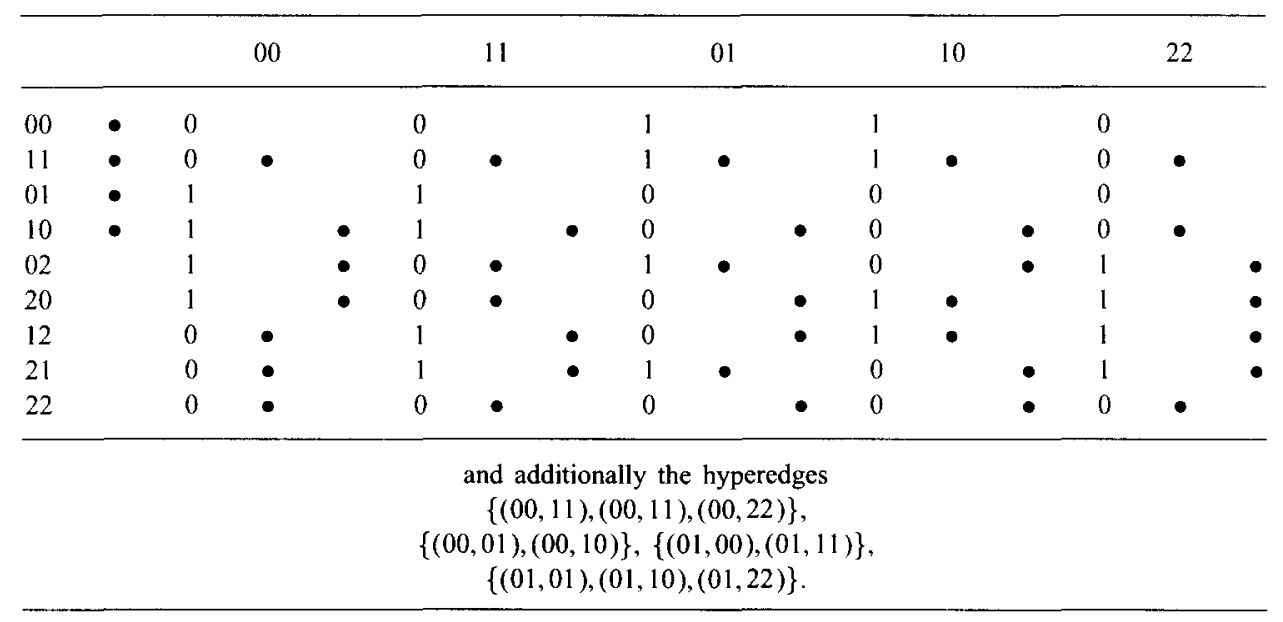

3. For this we have to use Table 2. Two points in one of the first three columns denote that the pair is in $I$. For example the first two points in the first column denote that 00 and 11 is a pair in $I$. The points in the other columns denote the squares. Here we have $\alpha \leqslant 6, \beta \leqslant 6$.

4. For this case we have to use Table 3 . Here we have $\alpha \leqslant 7, \beta \leqslant 6$.

5. $|\mathscr{S}(I)|=|\mathscr{S}(J)|=3: \alpha+\beta \leqslant 9$.

6. $\alpha \leqslant 8, \beta \leqslant 4$. This case needs Table 4 .

7. $\alpha \leqslant 6, \beta \leqslant 6$. Needed points are given to Table 5 .

8. $|\mathscr{P}(I)|=|\mathscr{S}(J)|=3, \alpha+\beta \leqslant 9$.

9. $\alpha \leqslant 7, \beta \leqslant 7$. Needed points are given in Table 6 .

10. This is the most complicated case. For this maximal matching pair we got $\alpha=\beta=8$, which is not good enough for our induction. We will discuss it later.

For the first 9 cases, we have $\alpha+\beta \leqslant 15$ and therefore by Eq. (3.1) and the induction hypothesis

$$
Q_{3}^{p}(n) \leqslant 15\left(2^{n-3}+1\right)^{2}
$$


For $n \geqslant 8$ we have

$$
Q_{3}^{p}(n) \leqslant 2^{2 n-2} .
$$

The induction works.

\subsection{The last case}

If we cannot find two positions $i, j$ such that $A_{s t}^{i j}, B_{s t}^{i j}$ are in one of the first 9 situations, then we prove the conjecture directly. Define

$$
A_{11}^{\prime}=A_{00}^{\prime}=A_{11} \cup A_{00} \quad \text { and } \quad A_{01}^{\prime}=A_{10}^{\prime}=A_{10} \cup A_{01} .
$$

Then

$$
A^{\prime}=\left(22 A_{22}\right) \cup\left(11 A_{11}^{\prime}\right) \cup\left(00 A_{00}^{\prime}\right) \cup\left(01 A_{01}^{\prime}\right) \cup\left(10 A_{10}^{\prime}\right)
$$

and the similarly defined $B^{\prime}$ are still a parity pair. Without loss of generality we assume for all $i, j$ the existence of a permutation of $(0,1,2)$, say $s_{0}, s_{1}, s_{2}$, such that $A_{s_{0} s_{0}}^{i j}=A_{s_{1} s_{1}}^{i j}, A_{s_{0} s_{1}}^{i j}=A_{s_{1} s_{0}}^{i j}$, and $A_{s_{0} s_{0}}^{i j}, A_{s_{0} s_{1}}^{i j}, A_{s_{2} s_{2}}^{i j}$ are disjoint. The other sets are empty. The same is also true for the $B_{s t}^{i j}$ 's. For $(i, j)=(1,2),(3,4), \ldots,(2 m-1,2 m), \ldots$, we can assume without loss of generality $s_{0}=0, s_{1}=1, s_{2}=2$. We claim that for any $(i, j)=(2 m-1,2 m)$

$$
\text { either } A_{22}^{i j}=\{\underline{2}\} \text { or } A_{22}^{i j}=\emptyset \text {. }
$$

If $n$ is even, an $(i, j)$ exists, say $(1,2)$, such that $(22, \ldots, 0, a, \ldots) \in A_{22}$, where the 0 is in position $i$. Then we have also $(22, \ldots, 1, \ldots) \in A_{22}$. Look at positions $(2, i)$, where $A_{20}^{2 i} \neq \emptyset$ and $A_{21}^{2 i} \neq \emptyset$.

If $A_{11}^{12}$ is not empty (otherwise, we can use induction), an element

$$
(11, \ldots, t, \ldots,)
$$

exists in $A_{11}^{12}$ and also an element

$$
(00, \ldots, t, \ldots,)
$$

exists in $A_{00}^{12}$, because $A_{11}=A_{00}$. If $t=0$, then $A_{10}^{2 i} A_{00}^{2 i}$ are not empty. This means that for position $(2, i) A_{s t}^{2 i}$ is not in the case 10 , because $A_{10}^{2 i}, A_{00}^{2 i}$, and $A_{20}^{2 i}$ are not empty. If $t=1$ we got $A_{11}^{2 i}, A_{01}^{2 i}$, and $A_{21}^{2 i}$ not empty, and thus we obtain the same conclusion. If $t=2$, we got $A_{20}^{2 i}, A_{21}^{2 i}, A_{02}^{2 i}$, and $A_{12}^{2 i}$ are not empty. This also contradicts the fact that we should be in the situation 10 . For $n$ even we proved that no element which has both 2 and non- 2 entries appears in $A$ or $B$. Then

$$
A^{\prime}=A \backslash A(22, \ldots, 2) \in\{0,1\}^{n} \quad \text { and } \quad B^{\prime}=B \backslash B(22, \ldots, 2) \in\{0,1\}^{n} .
$$


Since they have the same parity, if the parity is 0 , then

$$
\left|A^{\prime}\right| \leqslant 2^{n-1}, \quad\left|B^{\prime}\right| \leqslant 2^{n-1}, \quad|A| \leqslant 2^{n-1}+1 \text { and } \quad|B| \leqslant 2^{n-1}+1
$$

and if the parity is 1 , then $(22, \ldots, 22) \notin A \cap B$ and $|A| \leqslant 2^{n-1},|B| \leqslant 2^{n-1}$.

In fact, we have proved that, even in the case $n$ is odd, in the first $n-1$ positions, 2 and 0,1 never appear in the same element in $A$. We prove

$$
\left|\left\{x^{n} \in A: x^{n}=(22, \ldots, 2, s)\right\}\right|=1 .
$$

Otherwise the same argument will lead to the fact that $A_{s t}^{2 n}$ is not in the situation 10.

After a permutation for position $n$ we get the same result. However,

$$
\overbrace{(22, \ldots, 2)}^{n}
$$

can belong to only one of $A$ and $B$ in case $n$ is odd and $p=1$, and can belong to no one in case $n$ is odd and $p=0$. This proves the conjecture.

\section{References}

[1] R. Ahlswede, On code pairs with specified Hamming distances, Colloq Math. Soc. Janos 52 (1987) 9-47.

[2] R. Ahlswede, N. Cai, Z. Zhang, A general 4-words inequality with consequences for 2-way communication complexity, Adv. Appl. Math. 10 (1990) 75-94.

[3] R. Ahlswede, A. El Gamal, and K.F. Pang, A two family extremal problem in Hamming space, Discrete Math. 49 (1984) 1-5.

[4] R. Ahlswede, M. Moers, Inequalities for code pairs, European J. Combin. 9 (1988) 175-181.

[5] N. Cai, A bound of sizes of code pairs satisfying the strong 4-words property for the Lee distance, J. System Sci. and Math. Sci. 6 (1986) 129-135.

[6] P. Delsarte, P. Piret, An extension of an inequality by Ahlswede, El Gamal, and Pang for pairs of binary codes, Discrete Math. 55 (1985) 313-315.

[7] M. Deza, Une proprieté extrémale des plans projectifs finis dans une classe de code equidistants, Discrete Math. 6 (1973) 343-352.

[8] J.I. Hall, J.H. van Lint, Constant distance code pairs, Proc. Kon. Ned. Akd. v. Wet. (A) 88 (1985) $41-45$.

[9] H. van Lint, A theorem on equidistant codes, Discrete Math. 6 (1973) 353-358. 